\title{
Analysis of Characteristics of Rainfall in Tongchuan China During 50 Years
}

\author{
Li Yabin", Hou Kai, Qian Hui \\ Department of Environmental Science and Engineering, Chang'an University, Xi'an, China \\ Email address: \\ 893378831@qq.com (Li Yabin), 1123997326@qq.com (Hou Kai), 55480981@qq.com (Qian Hui) \\ *Corresponding author
}

\section{To cite this article:}

Li Yabin, Hou Kai, Qian Hui. Analysis of Characteristics of Rainfall in Tongchuan China During 50 Years. Science Discovery. Vol. 4, No. 3, 2016, pp. 176-182. doi: 10.11648/j.sd.20160403.14

Received: March 12, 2016; Accepted: April 15, 2016; Published: July 5, 2016

\begin{abstract}
According to the national weather service Tongchuan city for nearly 50 years (1964-2013) of rainfall measured data, the application of related theory and method of mathematical statistics, Mann-Kendall nonparametric tests, R/S analysis method, linear regression theory, all the methods are used to study these datas. Based on the results of mathematical statistics, analysis of the trends in Tongchuan city nearly 50 years of rainfall characteristics and time-space evolution. The results showed that the rainfall of Tongchuan is distributed unequal, it mainly concentrated in February, rainfall of Winter is lesser. In terms of the annual change, Tongchuan city nearly 50 years of rainfall overall showed a negative growth trend. At the same time, by using Mann-Kendall to Analysis this title we can see that, in 1, 2, 6, 7, 8, 11, 12 months, it showed rainfall increased, at the same time, the most obvious increase was in June. Analysis of the $\mathrm{H}$ index, in 1, 3, 4, 5, 6, 9 months they were greater than 0.5 , it was showed that rainfall time series with long-term sustainability, changes in future rainfall characteristics can still play an important role in today's rainfall predict. In this paper, China Tongchuan region for nearly 50 years of rainfall characteristics and trend of rainfall were studied, have discussed the changes in rainfall over the years as well as the possible future rainfall trends, base on this to guide the local economic construction, agricultural development and so on.
\end{abstract}

Keywords: Mann-Kendall Nonparametric Tests, R/S Analysis Method, Rainfall Characteristics, Rainfall Trend

\section{中国铜川市近50年降雨特征分析}

\author{
李亚斌, 侯凯, 钱会 \\ 环境科学与工程学院, 长安大学, 西安, 中国
}

\section{邮箱}

893378831@qq. com(李亚斌), 1123997326@qq. com（侯凯），55480981@qq.com(钱会）

摘要: 依据国家气象局铜川站近 50 年 (1964-2013) 的降雨实测数据, 应用数理统计的有关理论和方法、Mann-Kenda11
非参数检验法、 $R / S$ 分析法、一元线性回归等方法理论进行研究。基于数理统计结果, 分析了铜川市近 50 年的降雨特
征及趋势变化。研究结果表明: 铜川市年降雨量分布不均, 降雨量主要集中在 2 月, 冬季降雨量较少; 就年变化趋势而
言, 铜川市近 50 年的降雨量总体呈现负增长的趋势; 与此同时, 经Mean-Kenda11法分析可得, 在 $1 、 2 、 6 、 7 、 8 、 11$ 、
12 月份, 表现为降雨量增加, 同时 6 月份降雨量增加最为明显; $R / S$ 分析所得的 $H$ 指数, 在 $1 、 3 、 4 、 5 、 6 、 9$ 月份均
大于 0.5 , 表明这柴月份降雨量时间序列具有长期相关的持续性, 未来降雨的变化特征仍沿用目前整体降雨的变化趋势。 
本文对铜川地区近50年的降雨特征及降雨趋势进行了研究, 分析了降雨的多年变化情况以及未来可能的降雨变化趋势, 以此来为当地的经济建设、农业发展等提供依据。

关键词: Mann-Kendal1 非参数检验法, $\boldsymbol{R} / \boldsymbol{S}$ 分析, 降雨特征, 降雨趋势

\section{1. 引言}

全球气候变暖是最近 100 年以来气候变化方面最 显著的特征, 近50年变暖尤为明显, 降雨特征也在一 定程度上发生了变化 [1]，基于此，对降雨变化特征 的研究就显得尤为重要, 该做法不仅对地区的经济发 展有益，同时可以指导决策部门进行有关政策方针的 制定和实施 ${ }^{[2]}$ 。近年来，气候问题不断被提及，有关 降雨变化特征的研究层出不穷, 研究方法多种多样, 主要包括距平分析法、线性趋势分析法、 Mann-Kenda11 (M-K) 非参数突变检验法、Morlet (小 波分析法)、R/S分析等方法。2014年, 孙润等利用距 平分析法和线性趋势分析法研究半湿润半干旱典型 过渡区的定西温度、降水量变化特征发现: 定西市降 水变化存在着显著的区域差异, 总体降水幅度由东北 向西南呈增加趋势; 2013 年王翠柏等, 运用 Mann-Kenda11 非参数检验法和R/S 分析法研究永康 市近 50 年降雨时序变化特征发现: 永康市 $1 、 3 、 6 、 7 、$ 8、 $11 、 12$ 月这 7 个月的降雨量呈现上升的趋势, 其中, 8 月份降雨量的上升趋势较为明显; 其他 5 个月的降 雨量呈现下降趋势。上升和下降趋势都具有正的持续 性，会在未来继续保持，且 $1 、 6 、 8 、 12$ 月份降雨变 化趋势正的持续性很强。很多研究者都对自己所研究 的地区做了详细的结果分析, 同时也提出了很多建设 性意见, 但也有一些不足, 比如有些对于所研究地区 降雨量变化及趋势分析只是给出了一个合理推断, 不 能明确其相关的影响因子, 鉴于此, 本文将在研究的 基础上, 更加明确的指出其变化因子以及趋势。

中国铜川位于陕西省中部，渭河平原北部，交通便 利, 是关中经济带的重要组成部分, 地貌上形成南部、 西部低山与该区的残原梁峁。属大陆性季风气候, 四季 分明, 冬季寒冷, 夏季炎热, 春季升温较快, 秋季降温 迅速, 气温日较差大, 昼夜温差大, 气温月季变化明显。 因此, 研究区域的降水变化规律, 对了解气候变化特征, 农业的发展具有重要的现实意义。

\section{2. 数据来源、数据整理和研究方法}

在对问题进行研究之前, 我们首先要做一些准备工
作, 即对所研究问题的数据进行相关的说明, 如数据来 源, 数据整理以及在研究中所用到的研究方法, 下面将 对这三方面进行简单的介绍。

\section{1. 数据来源}

运用中国气象局, 陕西气象铜川站1964-2013年的 逐月降雨量资料, 铜川站所处位置为 $109^{\circ} 04^{\prime} \mathrm{E} 、 35^{\circ}$ $05^{\prime} \mathrm{N}$, 海拔978.9m。

\section{2. 数据整理}

根据中国陕西气象局铜川站所提供的1964-2013年 的降雨资料, 分析可得其50年的逐月降雨量变化及趋势 图（如图1所示），以此来初步表征其降雨特征和降雨 时序变化等内容。
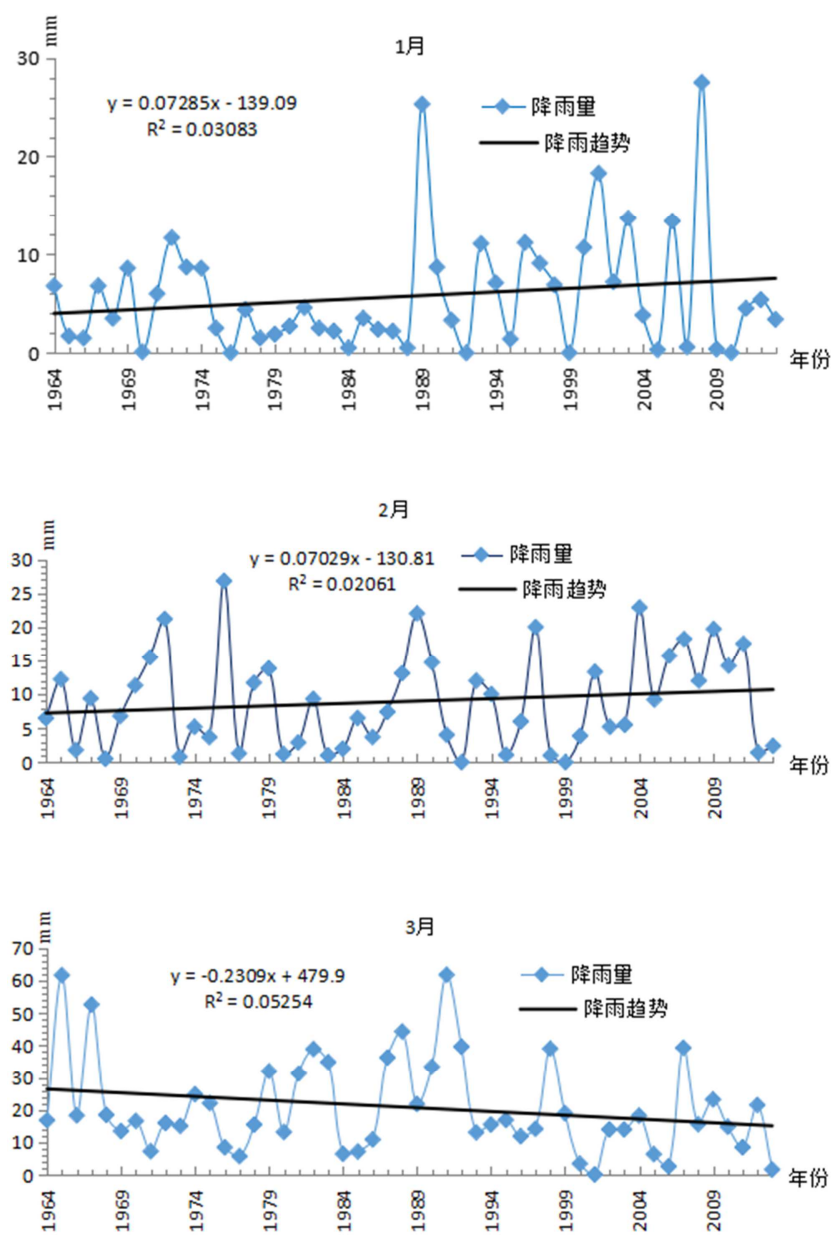

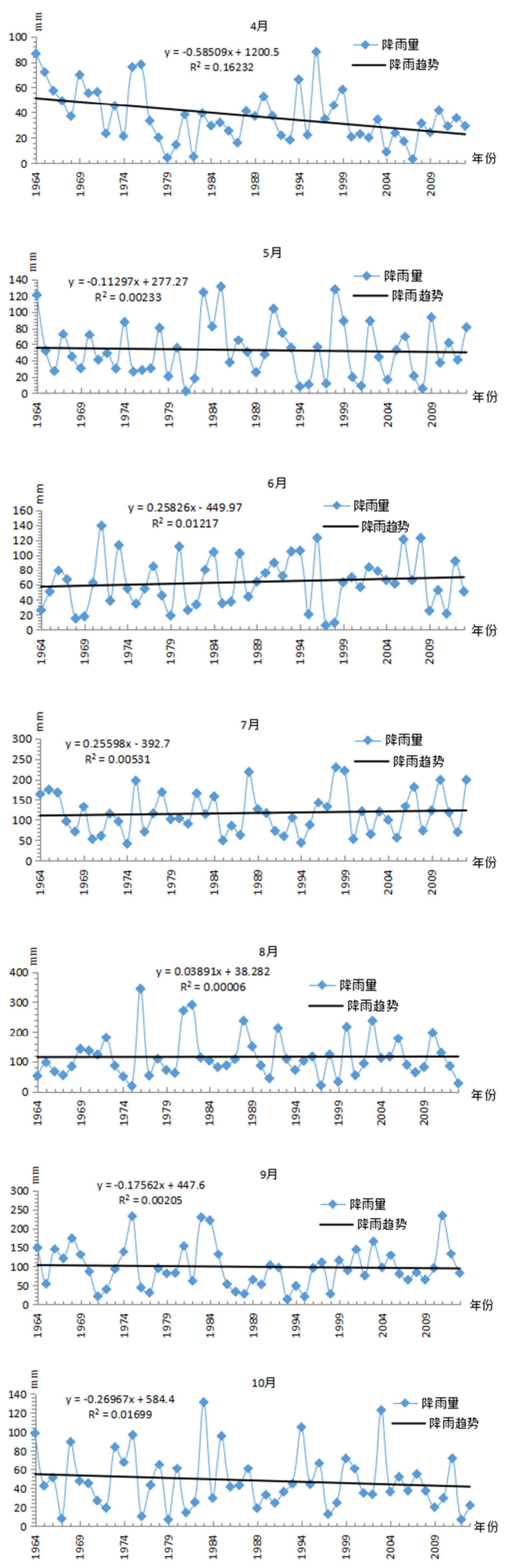
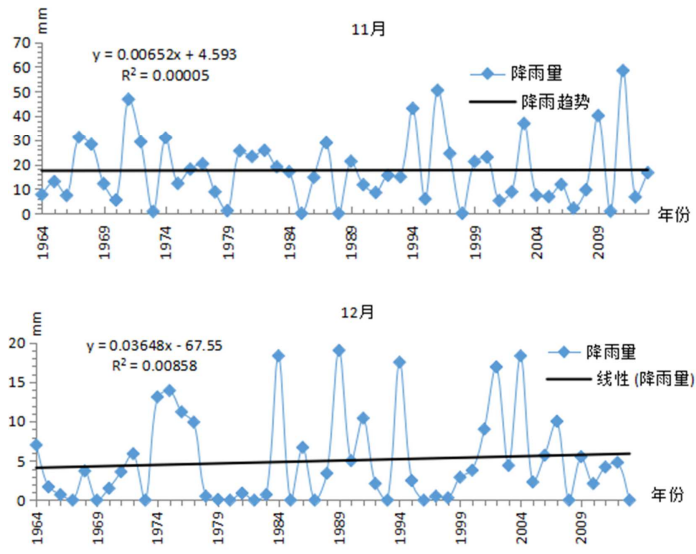

图 1 铜川市 1964-2013 年逐月降雨量变化及趋势图。

\section{3. 研究方法}

研究降雨量的时序变化特征方法有很多, 但本文采 用线性回归法、R/S、Mann-Kenda11法对其进行研究和 讨论。

\subsection{1. 回归分析法}

回归分析是研究数值型自变量与数值型因变量之 间的相关关系的一种统计分析方法, 回归分析按所处理 的变量多少为标准划分成一元回归分析和多元线性回 归分析, 一元回归分析是研究两个变量之间的相关关系; 同时, 按变量之间的关系形态为标准划分成线性回归和 非线性回归分析 [1-2]。其中本文重点使用一元线性回 归来分析降雨的时序变化特征。

\subsection{2. $\mathrm{R} / \mathrm{S}$ 分析法}

$R / S$ 分析方法可以区分具有长期非函数周期时间序 列, 其基本思想如下：给定一个时间序列 $\left(t_{1}, t_{2}, t_{3} \cdots t_{N}\right)$, 形成以固定的时间间隔 $\left(t_{2}-t_{1}=t_{3}-t_{2}=\cdots=t_{N}-t_{N-1}\right)$ 所对应的量 $\left(x_{i}\right), \mathrm{i}=1,2 \cdots \mathrm{N}$, 即 $x_{1}, x_{2}, x_{3} \cdots x_{N}$, 其中, 时间跨度为 $[3-5]$ :

$$
\Delta_{t}=t_{N}-t_{1}
$$

在时间跨度 $\Delta \mathrm{t}$ 内, 对应的量 $x_{i}$ 的平均值可以表示为:

$$
\overline{x_{N}}=\frac{\sum_{i=1}^{N} x_{i}}{N}, \quad \mathrm{~N}=1,2 \cdots
$$


依据式1、2可以得到以下三个关于时间序列的方程:

1）时间累积离差:

$$
x(i, N)=\sum_{k=1}^{i}\left(x_{k}-\overline{x_{N}}\right), 1 \leqslant \mathrm{i} \leqslant \mathrm{N} ;
$$

其中 $\mathrm{N}$ 代表变量 $x_{i}$ 在给定时间序列内的个数, $\mathrm{N}$ 随 着给定时间跨度的变化而发生变化; 当 N确定时, $x_{k}$ 也 就被确定, N的稳定性是其他一切统计计算的前提条件。

2) 时间序列极差:

$$
R\left(t_{N}-t_{1}\right)=R\left(\Delta_{t}\right)=\mathrm{R}=\max _{t_{1} \leq \leq t \leq t_{N}} x(t, N)-\min _{t_{1} \leq t \leq t_{N}} x(t, N)
$$

(其中, $R(N)$ 随着 $N$ 的增加而变化。)

3) 时间序列的标准差 (为了更形象的描述研究对 象之间的规律所提出的）;

$$
S=\left[\frac{1}{\Delta_{t}} \sum_{i=1}^{N}\left(x_{i}-x_{N}\right)^{2}\right]^{\frac{1}{2}}
$$

4) Hurst指数定义无量纲比R/S:

$$
\frac{R}{S}=\frac{\max _{t_{1} \leq t \leq t_{N}} x(t, N)-\min _{t_{1} \leq t \leq t_{N}} x(t, N)}{\left[\frac{1}{\Delta_{t}} \sum_{i=1}^{N}\left(x_{i}-x_{N}\right)^{2}\right]^{\frac{1}{2}}}
$$

此方法当用 $\lg (R / S) 、 \lg (N)$ 作为坐标轴时, 数据 组中的点大致呈线性关系（用最小二乘法拟合），由此 确定的斜率为Hurst系数，记作 $H$ ，根据 $H$ 的大小判 断时间序列的持续性, 判断时间序列未来的气候特征; 其中 $0 \leq H \leq 1$, 以 0.5 作为一个分界点, 当 $H=0.5$ 时, 时间序列的 $x_{i}$ 变化是无规律的, 具有完全随机性, 针 对目前降雨的变化特征对未来降雨不会产生影响; 当 $H=1$ 时, 称为完全预测围, 针对目前表示降雨将围绕 均值发生持续性的变化; 当 $0.5\langle H\langle 1$ 时, 说明时间序列 具有长期相关的持续性, 未来降雨的变化特征沿用以往 整体降雨的变化趋势, $H$ 越大, 其持续性越强, 跟以
往的关系越密切; 当 $0<H<0.5$ 时, 表明时间序列具有 与研究目标相反的持续性, 未来降雨的变化特征与以往 的降雨变化趋势呈现相反的趋势, $H$ 越趋近于 0 , 其相 反的趋势越强 $[6-7]$ 。

\section{3. 3. Mann-Kenda11非参数检验法}

Mann-Kenda11非参数检验法, 近年在时间序列的趋 势分析中被许多学者所应用, 其中应用于降雨量、气候 变化特征分析也越来越多。时间序列显著趋势分析法可 分为参数和非参数方法, 参数趋势测试需要独立和正态 分布的数据; 而非参数趋势测试只需要数据是独立的, 不需要遵从一定的正太分布, 也不受少数异常值的干扰, 更适用于顺序变量, 计算也比较简单。

Mann-Kenda11检验中，假设 $H_{0}$ 表示原时间序列 $\left(x_{1}, x_{2}, x_{3} \cdots x_{N}\right)$ 无变化趋势, $H_{1}$ 表示对应序列有 相应的趋势变化 $[8]$ 。具体如下所示:

给定的时间序列 $x_{i}$, 共有 $N$ 个样本, 构造统计量 $S$ :

$$
s=\sum_{i=1}^{N-1} \sum_{j=i+1}^{N} \operatorname{sgn}\left(x_{j}-x_{i}\right)
$$

式中：

$$
\operatorname{sgn}\left(x_{j}-x_{i}\right)=\operatorname{sgn}(h)= \begin{cases}1 & \left(x_{j}-x_{i}\right)=h>0 \\ 0 & \left(x_{j}-x_{i}\right)=h=0 \\ -1 & \left(x_{j}-x_{i}\right)=h<0\end{cases}
$$

在此式子中， $s$ 服从正太分布，根据正太分布的一 般性质可得 $s$ 的均值 0 , 方差 $\operatorname{Var}(s)=n(n-1)(2 n+5) / 18$; 则 $s$ 的统计检测值为 [10-10]:

$$
Z_{s}= \begin{cases}\frac{s-1}{\operatorname{Var}(s)} & s>0 \\ 0 & s=0 \\ \frac{s+1}{\operatorname{Var}(s)} & s<0\end{cases}
$$

该式子中, 当 $z_{s}>0$ 时, 表明序列变量呈现上升趋 
势; 当 $z_{s}<0$ 时, 表明序列变量呈现下降趋势; 对于给 定的置信区间a（显著水平），当 $\left|z_{s}\right|>\left|z_{1-a / 2}\right|$ 时，原 假设 $H_{0}$ 被拒绝, 表明时间序列有明显的上升或下降趋 势; 当 $\left|z_{\mathrm{s}}\right| \leq\left|z_{1-a / 2}\right|$, 原假设 $H_{0}$ 成立, 表明时间序列 没有明显的变化趋势 [11]。据此, 其变化趋势的大小可 用趋势系数 $\beta$ 表示:

\begin{tabular}{cll}
\hline 月份 & 降水量变化倾向率 $\mathrm{mm} / 10 \mathrm{a}$ & 方差 $\operatorname{Var}(s)$ \\
\hline 5 & -1.130 & 0.0023 \\
6 & 2.583 & 0.0122 \\
7 & 2.560 & 0.0053 \\
8 & 0.389 & $6 \mathrm{E}-05$ \\
9 & -1.756 & 0.0020 \\
10 & -2.697 & 0.0170 \\
11 & 0.065 & $5 \mathrm{E}-05$ \\
12 & 0.365 & 0.0086 \\
\hline
\end{tabular}

$$
\beta=\text { median }\left(\frac{x_{j}-x_{i}}{j-i}\right) \text {, 任意的 } \mathrm{j}>\mathrm{i}
$$

若 $\beta>0$, 表示呈上升趋势; 若 $\beta<0$, 表示呈下降 趋势 $[11]$ 。

\section{3. 结果与分析}

据统计数据1964-2013年逐月降雨量折线图和趋势 分析图(如图1)所示, 可得下表1(中国铜川市1964-2013 各月降水趋势分析）。

根据中国铜川当地气候变化特征, 结合实际情况, 有关部门对铜川市季节划分如下, 春季 $(3-5)$, 夏季 (6-8)，秋季（9-11），冬季（12-次年2月）。由表1 结合图1分析可得: 春季降雨量呈现明显减少的趋势, 但5月份降雨平均递减率较小; 夏季降雨量总体呈现较 为显著的增加趋势; 秋季降雨量表现为较为明显的减少 趋势; 冬季降雨量基本保持不变, 呈平稳状况; 降雨量 全年分配不均, 降雨主要集中在夏季, 且冬季降雨量占 全年降雨量比重较少。综合图2年降雨量变化趋势分析 图可得, 铜川市近50年的降雨量呈现负增长, 在 1984 年和 2004 年降雨量均出现较大幅度增加, 最大降雨量达 到了 $935 \mathrm{~mm}$; 在 1995 年其降雨量出现低谷, 为 $356 \mathrm{~mm}$, 仅 占最大降雨量的 $38.1 \%$ 。基于上述对铜川市的降雨分析, 对合理优化年内降水资源分配利用以及协调经济发展 具有重要的实际意义。

表1 中国铜川市1964-2013各月降水趋势分析。

\begin{tabular}{cll}
\hline 月份 & 降水量变化倾向率 $\mathrm{mm} / 10 \mathrm{a}$ & 方差 $\operatorname{Var}(s)$ \\
\hline 1 & 0.728 & 0.0308 \\
2 & 0.703 & 0.0206 \\
3 & -2.309 & 0.0525 \\
4 & -5.851 & 0.1623 \\
\hline
\end{tabular}

图2 1964-2013年中国铜川市逐年降雨量和趋势分析图。

\section{1. 降水量的变化趋势}

基于Mann-Kenda11检验法分析近 50 年的降水变化 趋势, 可得如下表所示的参数数据（统计检测值 $z_{s}$ 和 降雨趋势 $\beta$, 其中 $\beta$ 通过一元线性拟合（如图1趋势线 的斜率）所得。

在对中国铜川市近50年降雨量进行趋势检查时, 在 给定的 $\mathrm{a}$ 置信水平上, $z_{s}$ 的绝对值在大于等于 1.28 、 1. $64 、 1.96$ 和 2.32 时, 分别表示通过了可信度 $90 \%, 95 \%$, $97.5 \%, 99 \%$ 的显著性检验, 对应的a为 $0.2,0.1,0.05$, $0.02^{[12]}$ 。

由统计检测值 $z_{s}$ 和降雨趋势 $\beta$ 分析可得, $1 、 2 、 6$ 、 7、8、11、12月的 $z_{s}$ 值均大于 0 , 表明在这些月份降雨 量呈现了增加的趋势, 增加的幅度 (对应的降雨趋势 $\beta$ 的值）为 $0.065 \mathrm{~mm} / 10 \mathrm{a}$ 到 $2.583 \mathrm{~mm} / 10 \mathrm{a}$ 之间, 同时这些月 份对应的最大检测值 $z_{s}$ 为 2 月份的 1.10 ; 若在双边趋势 监测中, 设置信水平 $\mathrm{a}=0.05$ 时, 对应正太分布的临界值 为1.96[13-14], 而在 $z_{s}>0$ 时, 对应的最大值为 1.10 , 
且 $1.10<1.96$, 说明在这些月份内降雨没有明显的上升 趋势; 在 $3 、 4 、 5 、 9 、 10$ 月份，对应的统计检测值 $z_{s}$ 均 为负, 说明降雨量出现了不同程度的下降, 下降幅度介 于 $1.130 \mathrm{~mm} / 10 \mathrm{a}$ 到 $5.851 \mathrm{~mm} / 10 \mathrm{a}$, 且 $5.581>1.96$, 表明 有明显的下降趋势, 对应的月份为 4 月。

表2 铜川市1964-2013逐月降雨序列趋势参数。

\begin{tabular}{lll}
\hline 月份 & 统计监测值 $z_{s}$ & 降雨趋势 $\beta(\mathrm{mm} / 10 \mathrm{a})$ \\
\hline 1 & 0.54 & 0.728 \\
2 & 1.10 & 0.703 \\
3 & -1.46 & -2.309 \\
4 & -2.54 & -5.851 \\
5 & -0.37 & -1.130 \\
6 & 0.84 & 2.583 \\
7 & 0.49 & 2.560 \\
8 & 0.49 & 0.389 \\
9 & -0.22 & -1.756 \\
10 & -0.91 & -2.697 \\
11 & 0.70 & 0.065 \\
12 & 0.76 & 0.365 \\
\hline
\end{tabular}

\section{2. 降水量的持续性分析}

对降雨量进行持续性研究时, 通常根据 $R / S$ 所计 算得到的Hurst系数来表征降水的持续性, 据此各月份 降雨量序列的Hurst指数如下表3所示:

表3 铜川市1964-2013各月降雨量序列的Hurst指数。

\begin{tabular}{ll}
\hline 月份 & Hurst 指数 \\
\hline 1 & 0.77 \\
2 & 0.46 \\
3 & 0.54 \\
4 & 0.89 \\
5 & 0.64 \\
6 & 0.51 \\
7 & 0.41 \\
8 & 0.29 \\
9 & 0.71 \\
10 & 0.28 \\
11 & 0.28 \\
12 & 0.44 \\
\hline
\end{tabular}

由表可以清楚的看到, 在1、3、4、5、6、9月份, 降雨量序列的 $H$ 指数（好斯特指数）均超过了 0.5 , 表 明当前时间序列具有长期相关的持续性, 未来降雨的变 化特征沿用目前整体降雨的变化趋势, 且对于四月分的 $\mathrm{H}$ 指数 $(0.89)$ 比较大, 具有较强的的持续性; 而对于 2 、 $7 、 8 、 10 、 11 、 12$ 月份，其对应的 $H$ 指数均低于 0.5 , 表 明未来降雨的变化特征与目前的降雨变化趋势呈现相 反的趋势, 可能会出现一定的周期性的变化 [15]。

\section{4. 结论}

本文以中国铜川市1964-2013年降雨量数据为基础, 采用Mann-Kenda11法和 $R / S$ 分析法, 分析了铜川近50 年降雨量的时间变化特征及其变化趋势, 得出了如下结 论:

（1）铜川市近50年的降雨量呈现较为明显的递减, 且减少量为 $6.3 \mathrm{~mm} / 10 \mathrm{a}$; 这对该地区的农业发 展会产生一定的影响。因此, 针对铜川地区降 雨量呈现逐年递减的趋势, 政府及有关部门应 当做好相应的措施来应对因降雨量变化而带 来的问题, 如干旱等现象。

（2）根据Mann-Kenda11法获得的 $z_{s}$ 值，可以判定 在 $1 、 2 、 6 、 7 、 8 、 11 、 12$ 月的 $z_{s}$ 值均大于 0 , 表现为降雨增加的趋势, 这与采用一次线性回 归法所得结果是一致的; 降雨量增加相应的会 对该地区经济、农业等产生必要的推动作用, 合理利用降水, 充分发挥其作用显得尤为重要。

(3) 通过 $R / S$ 法对降雨量持续性分析得出, $H$ 指 数表现为有正有负, 降雨量在不同月份有其自 身的趋势变化特点, 其中, 在 4 月份的 $H$ 指数 （0.89）比较大，表现为很强的持续性。

（4）由于铜川地区年内降雨量分布不均, 降雨主要 集中在夏季, 冬季降雨稀少, 对此, 要提倡优 化当地降水利用, 采取夏季储存备用, 冬季取 用的原则，保证降雨的合理化分配及利用。

\section{致谢}

水利部公益性行业科研专项经费项目 (201301084)。

\section{参考文献}

[1] 孙润, 尚军林。定西市近47年气温、降水变化特征分析 [J], 陕西气象, 2015 (1)：11-14。 
[2] 钱会, 李培月, 吴建华。银川市近 49 a来降雨变化特征分 析 $[J]$, 华北水里水电学报, 2010 (4)，1-5。

[3] 赵华荣, 夏北成, 江学顶。桂林市近 50 年降雨变化特征 分析, 桂林理工大学学报 $[J], 2011$ (5) : 202-206。

[4] Jiang Zhou, Lihua Feng. Trend predictions in water resources using rescaled range $(\mathrm{R} / \mathrm{S})$ analysis, Environmental Earth Sciences[J], 2013, Vol.68 (8), pp. 2359-2363.

[5]王翠柏, 楼章华, 曹飞凤, 徐礼强。永康市近 50 年降雨量 时序变化特征分析 $[J]$ ，人民珠江，2013（2）：48-51。

[6] L. Acedo, Gilberto González-Parra, Abraham J. Arenas. An exact global solution for the classical S R/S epidemic model, Nonlinear Analysis: Real World Applications [J], 2009, Vol.11 (3), pp. 1819-1825.

[7] Juan Li, Yong Chen. Rescaled range (R/S) analysis on seismic activity parameters[J], Acta Seismologica Sinica, 2001, Vol. 14(2), pp. 148-155.

［8］曹洁萍, 迟道才, 武立强, 刘丽, 李帅莹, 于沝。Mann-Kenda11 检验方法在降水趋势分析中的应用研究 $[\mathrm{J}]$, 农业科技与装 备, 2008（10）：35-40。

[9] Sheng Yue, ChunYuan Wang. The Mann-Kendall Test
Modified by Effective Sample Size to Detect Trend in Serially Correlated Hydrological Series[J], Water Resources Management, 2004, Vol.18 (3).

[10] Milan Gocic . Analysis of changes in meteorological variables using Mann-Kendall and Sen's slope estimator statistical tests in Serbia[J], Global and Planetary Change, 2013(1), 172-182.

[11] 张涵丹, 卫伟, 薛䒹。基于 $\mathrm{R} / \mathrm{S}$ 分析和Mann-Kendal1检验的 定西市气温降水变化特征 $[J]$, 水土保持研究, 2015 (12): 183-189。

[12] Ying He, Aiying Wang, Haijing Huang. The trend of natural illuminance levels in 14 Chinese cities in the past 50 years, Energy[J], Sustainability and Society, 2013, Vol. 3 (1), pp. 1-11.

[13] 康淑媛, 张勃, 柳景峰, 杨明金。基于Mann-Kendall法的 张掖市降水量时空分布规律分析 [J], 资源科学, 2009 (3): 501-507。

[14] 张燕明, 文俊, 王新华, 刘粀。基于Mann-Kendall分析的 昆明降雨与气温变化趋势研究 $[J]$, 安徽农业科学, 2011, 39 (25) : 15755-15757.

[15] 韩海波。Hurst指数及其在exce1中的实现 [M], 数量经济研 究，2006年第4期。 